\title{
Fuzzy Feature Visualization of 3D Vector Field by Information-Entropy-Based Texture Adaptation
}

\author{
Huaihui Wang, Huaxun Xu, Liang Zeng and Sikun Li
}

School of Computer, National University of Defense Technology, Changsha 410073, China

\begin{abstract}
Texture adaptation is a challenging issue in texture-based feature visualization. In order to visualize as more information as we can, this paper presents a texture adaptation technique for fuzzy feature visualization of 3D vector field, taking into account information quantity carried by vector field and texture based on extended information entropy. Two definitions of information measurement for 3D vector field and noise texture, MIE and RNIE, are proposed to quantitatively represent the information carried by them. A noise generation algorithm based on three principles derived from minimal differentia of MIE and RNIE is designed to obtain an approximately optimal distribution of noise fragments which shows more details than those used before. A discussion of results is included to demonstrate our algorithm which leads to a more reasonable visualization results based on fuzzy feature measurement and information quantity.

Index Terms - Scientific visualization; vector field; texture adaptation; fuzzy feature extraction; extended information entropy;
\end{abstract}

\section{INTRODUCTION}

Vector field visualization plays an important role in scientific research and is widely used to analyze data originating from numerical simulations or measurements such as those of computational fluid dynamics (CFD), climate modeling, and electromagnetism. Normally, these data is too confused to analyze directly and should be processed in some efficient approaches such as visualization methods. There are many ways to visualize vector data, among which texture-based visualization is a promising one since it can globally represent the information carried by those data.

Line integral convolution (LIC) [1] is one of the most important approaches of texture-based visualization. There are several advantages using LIC to visualize vector field, including high image quality, easy to accelerate using GPU and so on, which make LIC a most popular method for $2 \mathrm{D}$ vector filed visualization.

There are several challenges for texture-based visualization when extended to 3D vector fields, one of which is the problem of occlusion. Occlusion is the nature of 3D visualization and could be eased by several approaches such as interactive anal ysis, animation and feature-based visualization. Feature-based visualization seems more promising due to its ability to distinguish the most valuable part of a flow field data set from uninterested data. Many feature detectors have been developed such as $\lambda_{2}$-criterian for vortex, Non-local vortex detector and $\mathrm{M}_{\mathrm{z}^{-}}$criterian for vortex. Occlusion and clutter are eased by feature extraction and the corresponding visualization methods such as focus-and-context visualization that highlights important areas and ignores irrelevant regions. One major issue that is neglected by many researchers is how to measure the information of a vector field and the information differentia between the vector field and its final display image which lead to an improper representation of vector field. Typically, one could make the context of a vector field transparent while it carries some information of the vector field, though the context is not so important to the feature representation. On the other hand, feature area may be disposed with so many texture fragments in order to cover every detail of the feature, which makes feature area so occluded and complicated to analyze. Therefore, we need an information measurement to adapt the distribution of texture fragments, using which the final image would carry as much information as it could.

This paper proposes an effective method for information measurement based on extended information entropy. First, we obtain the membership degree of a 3D vector field using fuzzy feature detector [2][3], which indicates the fuzzy membership of every vertex in this vector field. Second, two extended information entropy, MIE and RNIE are proposed to measure the information carried by this fuzzy measurement field and the noise texture field generated from it. Third, an approximately optimal distribution of noise fragments with the most information can be generated using noise generation algorithm based on three principles derived from the minimal differentia between MIE and RNIE.

This paper improves the work of Xu et al. [2][3] in the following ways: First, several concepts of information measurement are proposed to determine the distribution of texture. Second, a texture adaptation algorithm is added in order to obtain a visualization result with maximum information defined by the above concepts. 


\section{RELATED WORK}

Texture-based visualizations are an important part for vector field analysis. The first attempt to utilize texture for visualization is spot noise [4] and line integral convolution (LIC) [1], and then a method using moving textures was introduced to the visualization community [5]. Based on this work, 2D Lagrangian-Eulerian Advection (LEA) [6] and Image-Based Flow Visualization (IBFV) [7] were developed by researchers. 2D LIC for steady vector field was extended by Shen et al. to UFLIC for 2D unsteady vector field [8]. 2D Texture-based methods can be extended to 3D vector field. 3D IBFV [9] was presented by Telea et al., and a method for 3D unsteady vector field visualization using real-time advection and volumetric illumination was developed by Weiskopf et al. [10][11]. Falk et al. proposed an output-sensitive 3D line integral convolution method to visualize 3D vector field [12]. All these 3D texture-based method utilized feature detectors to reduce uninterested flow regions and highlight important flow areas.

Feature detectors are designed to extract important structures in flow field from flow field data set. In the analysis of flow field, features such as critical points, vortices and certain typical lines play a crucial role. Based on these concepts, Helman and Hesselink first introduced vector field topology to the visualization community [13], and over two decades topology-based visualization has achieved a high level of proficiency in $2 \mathrm{D}$ planar steady flow fields and a great improvement in unsteady flow fields. A comprehensive overview on feature-and-topology-based visualization is given in the survey article [14]. To extract vortex structures, a local criterion was proposed by Jeong and Hussain [15]. A system that could analyze vortex structures contained in turbulent flows by means of a novel vortex core line detection method was presented by $\mathrm{S}$. Stegmaier et al. [16]. Recently, an objective definition of a vortex was given by G. Haller, which was derived from Lagrangian considerations, but used Eulerian quantities [17]. The paper also proposed Mz-criterian for vortex extraction. H. Janicke proposed a method using a local statistical complexity approach to automatically detect distinctive structures in time-dependent multi-fields [18]. T. Schafhitzel gave a vortex core line extraction technique based on vortex region criterion to detect vortex features in 3D flow field [19]. P. Christoph proposed a new method to visualize two-dimensional vortex regions using hierarchical representation induced by swirling motion. In his work, a new definition of vortex region was given as closed loops that intersect the flow field at a constant angle, and the author also presented a parameter free algorithm for identification of these regions [20]. A fuzzy feature extraction method based on fuzzy theory was designed by Hua-Xun $\mathrm{Xu}$ which defined flow feature as a fuzzy partition of vector field [2][3].

Information entropy has already been used in visualization area to enhance the rendering results. Ming-Yuen Chan proposed an entropy-based technique for quality enhancement of direct volume rendered images [21], which utilize an extended version of information entropy for information measurement of images and rays. The author takes a quantitative way to meas- ure information in visualization results, makes the conclusion more convincing.

Our work relies heavily on LIC [12] and fuzzy feature detector [2][3]. We use GPU to realize LIC computation which leads to an interactive rendering efficiency, and fuzzy feature measurement field is used to generate a noise fragments distribution.

\section{INFORMATION MEASUREMENT}

Information measurement is built upon the results of fussy feature extraction. In this section, we will shortly introduce the mathematical description of 3D flow features using fuzzy theory proposed in [2][3] firstly, and then give the information measurement of vector field and noise distribution.

\subsection{Fuzzy Feature Extraction}

The use of augmented reality systems in educational settings, per se, is not novel. Shelton and Hedley [6] describe a research project in which they used augmented reality to help teach undergraduate geography students about earth-sun relationships. They examined over thirty students who participated in an augmented reality exercise containing models designed to teach concepts of rotation/revolution, solstice/equinox, and seasonal variation of light and temperature, and found a significant overall improvement in student understanding after the augmented reality exercise, as well as a reduction in student misunderstandings.

Based on fuzzy theory, flow feature in a flow is defined as a fuzzy subset $F_{i}$, which has one special attribute $i$ in the 3D flow $\mathrm{X}$. The mapping $\mu_{\mathrm{F}}^{\mathrm{i}}(\mathrm{x})$ is a membership function, which maps $\forall \mathrm{x} \in \mathrm{X}$ to a certain value in the range $[0,1]$, named as membership degree of $\mathrm{x}$ for feature subset. When a position $\mathrm{x}$ get a greater value $\mu_{\mathrm{F}}^{\mathrm{i}}(\mathrm{x})$, it means that the probability of this point subject to the feature region is larger. Feature region with $\mathrm{i}$ property is defined as a crisp set containing all points meeting $\mu_{\mathrm{F}}^{\mathrm{i}}(\mathrm{x})>0.5$ in flow domain $\mathrm{U}$, and the feature kernel is correspondingly defined as a subset of it with $\mu_{\mathrm{F}}^{\mathrm{i}}(\mathrm{x})=1$.

This definition of flow feature based on fuzzy theory is more advantageous than others due to its qualitative description for the flow feature. For instance, although we do not understand vortex feature clearly, we know that if a position gets a greater $\lambda_{2}$ value, the probability it is in the vortex region is greater.

Fuzzy rules are used to determine whether a point in flow field is in a special feature region or not. Based on the definitions introduced above, relevant fuzzy rules are built to divide the whole field into feature regions and context. There are several rules to describe flow features such as $\lambda_{2}$ rule, Eulerian distance rule, pressure rule, etc. This method is flexible for adding new rules to feature extraction system without changing its basic framework, which seems more promising to other feature extraction framework.

A fuzzy k-means-like method was proposed to calculate feature regions based on the established rules. Firstly, a characteristic vector for each sample point in 3D flow fields is built according to relevant rules. Finally, the membership degree of 
each point is computed by the formula referred by [2] in order to achieve minimal characteristic vector differentia. This approach can be proved to be optimal in minimum-square-sum rule.

\subsection{Membership Degree Visualization}

Textures generated by existing methods distribute either uniformly in the vector field or controlled by some focus-and-context algorithm such as the above multi-resolution controller. Uniformly distributed textures can not represent the full detail of a 3D vector flow field. With dense representation, information will be hided to some extent. On the other hand, sparse representation may lose some important feature information. Although measurement field calculated by fuzzy feature detector can easily control texture distribution and generate a focus-and context effect, the final images is not so satisfying. As is shown in Fig. 1, it seems not suitable to treat the pure feature measurement $\mathrm{M}[\mathrm{P}]$ as the probability of texture distribution, and the input texture controlled by a threshold loses some information.

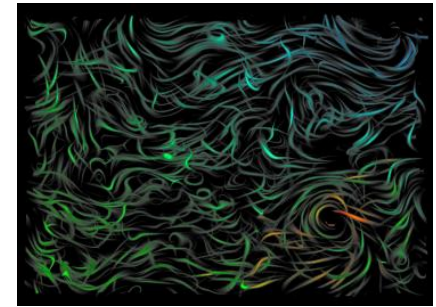

(a)

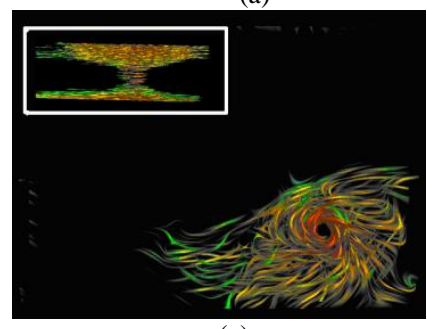

(c)

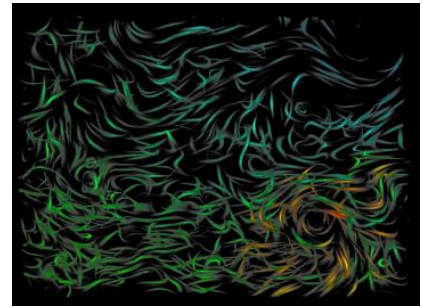

(b)

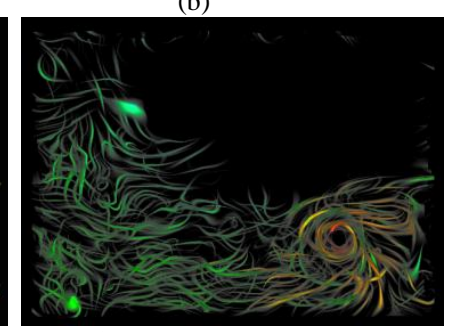

(d)
Fig. 1. Visualization results of hurricane Haitang data using feature extractor. (a) Uniformly distributed sparse texture. (b) Take the pure feature measurement as the probability of texture distribution. (c) Texture controlled by a threshold $\mathrm{a}=0.5$ for $\mathrm{r}^{*} \mathrm{M}[\mathrm{P}]$. (d) Texture controlled by $\mathrm{a}=0.25$ (Color Plate 7)

One shortcoming of this multi-field resolution method is the difficulty to achieve a reasonable noise texture distribution. Pure feature measurement does not merely represent the probability of existed texture appeared at the point, it carries more information. Although the feature area is represented in high detail in Fig. 1 (d), the context takes more information than that is displayed in the final image. It means that some texture fragments need to be deployed at the context area in order to show the context information. On the other hand, too many texture fragments are placed at the feature region to represent the feature as is shown in Fig. 1 (c), which could be expressed by fewer texture fragments with the same information represented in the image. So a reasonable noise texture distribution is difficult to attain, and needs many experiments for the threshold or other adjustment.
To address these problems, this paper proposes a rational measurement for vector field and noise texture, and presents a noise generation algorithm based on minimal differentia between them.

\subsection{Information Measurement for Vector Field}

Information entropy is the basic concept in information theory, which can measure information quantity in a mathematical way. Suppose that we have a set of probabilities (a probability distribution) $\mathrm{P}=\left(\mathrm{p}_{1}, \mathrm{p}_{2}, \ldots, \mathrm{p}_{\mathrm{n}}\right)$, the entropy of the distribution $\mathrm{P}$ is defined by:

$$
H(P)=\sum_{i=1}^{n} p_{i} * \log \left(1 / p_{i}\right)
$$

$\mathrm{H}(\mathrm{P})$ is the measure of the average information carried by the probability distribution. The larger $\mathrm{H}(\mathrm{P})$ is, the more disordered this random process is, and the more information is needed to fully understand this random process. So we draw the conclusion that the information carried by random process $\mathrm{P}$ is in inverse proportion to $\mathrm{H}(\mathrm{P})$. In the frame work of vector field information measurement which is different from pure random event, every point carries information in itself. Generally speaking, points in a certain feature region represent much more information than those far away from the feature. So we use an extended version of information entropy to measure the information contained in vector field. First, we assume that fuzzy feature measurement field generated by fuzzy feature extractor represents the information of each point in the corresponding vector field. This makes sense due to the definition of fuzzy feature and the principle that points near the feature region carry more information than those far from it. The larger the membership degree (fuzzy feature measurement) is, the smaller the distance between this point and the feature kernel, and the more information this point carries. Second, the average information of points in vector field can be measured as:

$$
H(V)=\sum_{i=1}^{n} \Phi\left(m_{i}\right) p_{m_{i}} * \log \left(1 /\left(\Phi\left(m_{i}\right) * p_{m_{i}}\right)\right)
$$

Where $m_{i}$ is the value of membership degree, $p_{m i}$ is the percentage of points with membership degree mi to the total, $\Phi\left(\mathrm{m}_{\mathrm{i}}\right)$ is the weight mapping function of membership degree, $\mathrm{n}$ is the count of levels into which the membership value is divided. We name $\mathrm{H}(\mathrm{V})$ Membership Information Entropy (MIE).

There are several properties of $\mathrm{H}(\mathrm{V})$ that are important for the application of this extended version of information entropy.

Property 1: The larger $\mathrm{H}(\mathrm{V})$ is, the less information the vector field possesses. This is identical to information entropy which is a type of minus entropy flow.

Property 2: $\Phi\left(\mathrm{m}_{\mathrm{i}}\right)$ maps larger $\mathrm{m}_{\mathrm{i}}$ to a smaller value that indicate larger information quantity. This is derived from property 1 and the definition of information entropy, which represents the disorder of a random process.

With the definition of MIE we can easily measure the information within a vector field.

\subsection{Information Measurement for Rendered Noise}

Following the definition of MIE, we will design another in- 
formation measurement for the multi-resolution noise texture to be rendered using LIC. Unlike vector field, the multi-resolution noise texture carries visual information in itself, which would be influenced by occlusion between each other. The measurement of noise should take occlusion effect as an impact factor for measurement calculation.

When a texture fragment is occluded by another one along the view direction, the information of it is reduced to some extent. As the texture fragments ahead get more, the information of this texture fragment becomes smaller. We can use an occlusion factor $\Psi$ (x) defined in formula (4) to represent the impact of occlusion.

We use the following formula to calculate the information measurement for the multi-resolution noise texture which is named as Rendered Noise Information Entropy (RNIE).

$$
\begin{aligned}
H(X)= & \sum_{i=1}^{n}\left\{\Phi\left(m_{i}\right) p_{i} \log \left(1 /\left(* \Phi\left(m_{i}\right) p_{i}\right)\right)\right. \\
& \left.*\left[\sum_{j=1}^{n_{i}} \Upsilon\left(\Psi_{m_{i}}\left(x_{i j}\right)\right)\right] / n_{i}\right\}
\end{aligned}
$$

Where $n_{i}$ is the count of noise texture fragment, whose membership degree is $m_{i}, Y(x)$ is a degressive function that maps information measurement to disorder degree in order to keep the inherent property of information entropy. $\Psi(x)$ is occlusion impact function which maps noise texture fragment $\mathrm{x}$ to a value that indicates its information contribution to the final image, and it is defined as:

$$
\Psi(x)=\left\{\begin{array}{cc}
1 & \text { if } x \text { is not occluded } \\
\Psi\left(x^{\prime}\right) \cdot f & x \text { is occluded by } x^{\prime} .0<f<1
\end{array}\right.
$$

Where $\mathrm{f}$ is a constant factor that indicates the information attenuation due to occlusion, $x^{\prime}$ is the texture fragment that occludes x directly.

RNIE is used to measure the information of the final rendered noise along some view direction. Each component of MIE is multiplied with an occlusion factor, whose physical meaning is adapted to information entropy. That is to say, occlusion factor indicates the disorder degree of occluded membership.

\subsection{Optimal Noise Distribution}

The objective function for optimizing noise distribution $\Omega$ is the differentia of MIE and RNIE, and we can obtain the optimal noise distribution $\Omega *$ by solving this equation:

$$
\begin{aligned}
\Omega^{*} & =\underset{\Omega}{\arg \min } E_{H} \\
& =\underset{\Omega}{\arg \min }|H(X)-H(V)|
\end{aligned}
$$

Assume that $\mathrm{n}$ is the count of texture fragments ahead of $\mathrm{x}_{\mathrm{ij}}$, $\mathrm{n}_{\max }$ is the maximum value of $\mathrm{n}$ that is allowed in this visualization system, we define these two mapping function $\Phi\left(\mathrm{m}_{\mathrm{i}}\right)$ and $\mathrm{Y}\left(\Psi_{\mathrm{mi}}\left(\mathrm{x}_{\mathrm{ij}}\right)\right)$ in our visualization system as follows:

$$
\Phi\left(m_{i}\right):=\left\{\begin{array}{cc}
1 / m_{i}^{3} & 0 \leq m_{i}<0.5 \\
1 / \sqrt{m_{i}} & 0.5 \leq m_{i} \leq 1
\end{array}\right.
$$

$$
\begin{aligned}
& \Upsilon^{\prime}\left(\Psi_{m_{i}}\left(x_{i j}\right)\right)=\left\{\begin{array}{cc}
1 / f^{10 n / n_{\max }} & n \leq n_{\max } \\
\infty & n>n_{\max }
\end{array}\right. \\
& \Upsilon\left(\Psi_{m_{i}}\left(x_{i j}\right)\right)=\left\{\begin{array}{cc}
\Upsilon^{\prime}\left(\Psi_{m_{i}}\left(x_{i j}\right)\right)^{0.1} & 1 / \Phi\left(m_{i}\right)>0.5 \\
\Upsilon^{\prime}\left(\Psi_{m_{i}}\left(x_{i j}\right)\right)^{4} & 1 / \Phi\left(m_{i}\right) \leq 0.5
\end{array}\right.
\end{aligned}
$$

The optimal noise distribution $\Omega *$ depends on view direction, different direction leads to different distribution. Based on these definitions we proposed a noise generation algorithm to obtain an approximate optimal noise distribution.

\section{ALGORITHM DESIGN}

In this section, we propose an algorithm to get an approximate optimal noise distribution. Derived from the above definitions, we obtain several principles for noise distribution in order to make EH almost reach its minimum. Noise generation is a random process, whose probability distribution complies with the principles follows. The noise fragments generated by this algorithm represent information that should be visualized, which is contrary to information entropy.

\subsection{Noise Distribution Principle}

It is difficult to get the optimal noise distribution directly by mathematical method, so we design three principles to control the random process of noise generation, taking into account every impact factor defined in MIE and RNIE.

Principle 1: Membership degree is an impact factor for noise fragment generation probability. Membership degree is sign of important feature. High membership degree means high probability of this point to be the feature extracted. So it needs to be visualized in more detail. On the other hand, low membership degree must not. Therefore, $F_{m e m}=1 / \Phi\left(m_{i}\right)$ is designed in membership degree perspective to control the probability of point with membership degree mi.

Principle 2: High probability of data points with the same membership degree is an impact factor for noise fragment generation probability. In information theory, an event with high probability means high information or less disorder. So points with high probability membership degree need to be visualized in more detail, and the lower ones in less detail. Percentage factor $\mathrm{F}_{\mathrm{pro}}=0.25^{*} \mathrm{p}_{\mathrm{M}(\mathrm{p})} /\left(\mathrm{p}_{\max }-\mathrm{p}_{\min }\right)$ is used to represent the percentage impact discussed in information theory. Although we treat noise fragments as a random process, the noise field is not quite the same with random process discussed in information theory, so we put the range 0.25 to this percentage factor in order to reduce the impact of it.

Principle 3: Points that are occluded by other noise fragments should have low probability to generate noise fragment. It is obvious that noise fragment that is occluded by some others carries less information than those which is not. So positions that are occluded should have low probability to generate noise fragments. Therefore, an occlusion factor $F_{\text {occ }}=1 / Y\left(\Psi_{m i}\left(x_{i j}\right)\right)$ is multiplied to the control factor. 


\subsection{Noise Generation Algorithm}

According to the definitions of MIE and RNIE and the three principles above, we know that the noise generation probability of a random position should be controlled by three factors: membership degree, percentage of this membership degree and occlusion impact. In order to make the differentia of MIE and RNIE nearly reaches its minimum, noise fragment percentage pi should be as closer to membership degree percentage $\mathrm{p}_{\mathrm{mi}}$ as possible. And the occlusion factor $\Psi(\mathrm{x})$ must be as closer to 1 as possible, that is to say, every noise fragment should not be occluded by others. So the generation probability of positions that is occluded must be lower than that is not.

Algorithm: Noise Generation

Input: Membership degree field $\mathrm{M}(\mathrm{P})$, Halton series $\mathrm{H}(\mathrm{x})$, maximal noise fragment count $\mathrm{N}$.

Output: Approximate optimal distributed Noise N(x).

(1) Initialize $\mathrm{N}(\mathrm{x})$.

(2) WHILE (count of generated noise fragments $n<N$ )

(3) Generate a random position $\mathrm{p}$ in flow area.

(4) Get the membership degree $M(p)$ of $p$.

(5) Generate a random value $\mathrm{r} \in[0,1]$.

(6) Scan the noise fragment ahead of $\mathrm{p}$ and get the count of them $\mathrm{n}_{\text {occ }}$.

(7) Calculate membership degree factor $F_{m e m}=1 / \Phi\left(m_{i}\right)$.

(8) Calculate the percentage $\left.\mathrm{p}_{\mathrm{M}(\mathrm{p}}\right)$ of membership degree $\mathrm{M}(\mathrm{p})$, and membership degree probability factor $\mathrm{F}_{\mathrm{pro}}=$

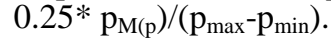

(9) Calculate occlusion factor $\mathrm{F}_{\mathrm{occ}}=1 / \mathrm{Y}\left(\Psi_{\mathrm{mi}}\left(\mathrm{x}_{\mathrm{ij}}\right)\right)$.

(10) IF $\left(\mathrm{r}<\left(\mathrm{F}_{\text {mem }}+\mathrm{F}_{\text {pro }}\right)^{*} \mathrm{~F}_{\text {occ }}\right)$

(11) FOR (Each grid cell $\mathrm{x}$ )

(12) $\quad \mathrm{N}(\mathrm{x})=$ GaussFilter $(\mathrm{H}(\mathrm{x}))$.

(13) END FOR.

(14) n++.

(15) END IF.

(16) END WHILE.

In the above algorithm, we use a random process to control the distribution of noise fragments. The three factors that impact the probability of noise fragment generation are derived from the principles discussed in section 4.1.

\section{RESULTS AND ANALYSIS}

In this section, we take vector field data of hurricane Haitang as an example to illustrate the effectiveness of our noise generation algorithm. Several visualization results are shown in Fig. 2, (a) is the rendering result of noise texture using threshold 0.25 to $\mathrm{r}^{*} \mathrm{M}[\mathrm{P}]$, (b) is the rendering result of noise texture using membership degree factor $F_{\text {mem }}$ only, (c) adding membership degree probability factor $F_{\text {pro }}$ to (b), (d) taking all these three factors, $F_{\text {mem }}, F_{\text {pro }}$ and $F_{\text {occ }}$, into account.

Fig. 2 compares the two approaches of threshold and control factors proposed by this paper. With membership degree factor $\mathrm{F}_{\text {mem }}$, the feature region is highlighted by enough noise fragments, as is shown in (a), and the result is better than using threshold [2][3] only, which is shown in Fig. 1 (c)(d). Fig. 2 (c) carries more information than Fig. 2 (b), which can be seen from the rendering images directly. Some noise fragments are

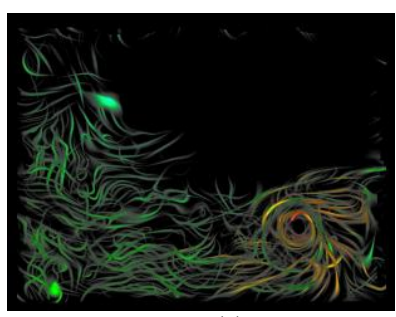

(a)

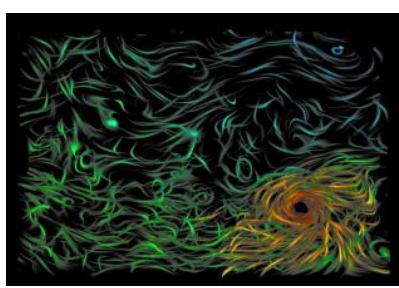

(c)

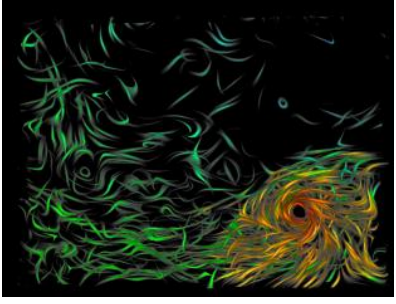

(b)

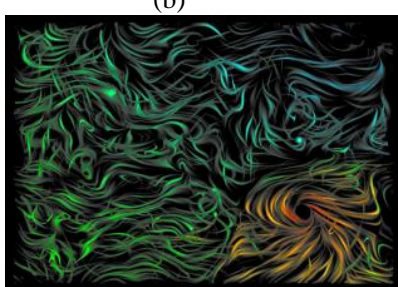

(d)
Fig. 2. Visualization results of hurricane Haitang data (a) Texture con trolled by a pure threshold $\mathrm{a}=0.25$ for $\mathrm{r}^{*} \mathrm{M}[\mathrm{P}]$. (b) Using membership degree factor only. (c) Controlled by both membership degree factor and membership degree probability factor. (d) Take all three factors into account.

distributed to regions with low membership degree, which leads to a better representation of the vector field than (b) because of more rendering details. The feature region is also deployed with enough noise fragments to fully represent the details of vortex core. (d) is the result taking all three factors into account, and we can see that the noise fragments that are not occluded by others are more than other three images, which means more detail is shown in (d) with equal noise fragments.

We further analyze the distribution of noise fragments in the following ways. First, the average noise fragments deployed to each point in vector field are calculated and shown in Fig. 3. We can see that noise distribution with factor $F_{\text {mem }}$ only gives the best distribution in principle that high membership degree needs more noise fragments. It makes sense that $\mathrm{F}_{\text {mem }}$ take only membership degree into account. Although noise distribution with factor $F_{\text {mem }}$ and $F_{\text {pro }}$ or with $F_{\text {mem }}, F_{\text {pro }}$ and $F_{\text {occ }}$ are not so optimal in membership principle due to occlusion or probability consideration, they are more proper than the distribution with threshold 0.25 .

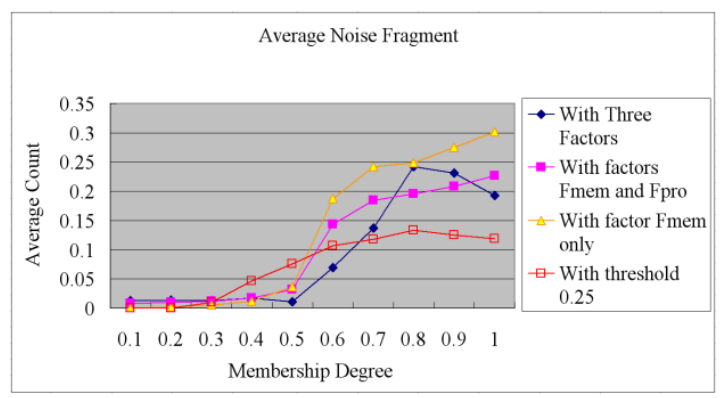

Fig. 3. Distribution of average noise fragment on membership degree. (a) With all three factors proposed in this paper, $F_{m e m}, F_{\text {pro }}$ and $F_{\text {occ. }}$ (b) With factors $F_{m e m}$ and $F_{\text {pro. }}$ (c) With factor $F_{\text {mem }}$ only. (d) With threshold 0.25 to $\mathrm{r}^{*} \mathrm{M}[\mathrm{P}]$

Secondly, the distribution of noise fragments along view direction is shown in Fig. 4, which strongly demonstrates the 
effect of occlusion factor. Nearly 70 percent noise fragments are distributed within 100 grids along the view direction depth. More information of the region near the viewer is shown due to the distribution with occlusion factor. Compared with the distribution without occlusion factor and on average membership degree, the superiority of occlusion factor is obvious. Fragment distribution on average membership degree is the noise fragments distributed on the average membership degree of each plane perpendicular to view direction.

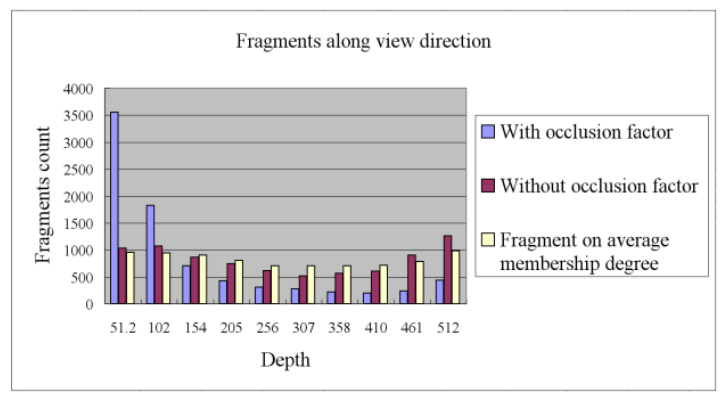

Fig. 4. Distribution of noise fragment along view direction. (a) With occlusion factor. (b) Without occlusion factor. (c) Fragments on average membership degree.

Finally, the differentia of MIE and RNIE is calculated and shown in Fig. 5. MIE is calculated from vector field, so it is a constant value when vector field is constant. The MIE of hurricane Haitang is 1.496 , so only RNIE is calculated and shown in Fig. 2. It is obvious that RNIE with occlusion factor is much lower than the other two methods, and this means more information is displayed with occlusion factor.

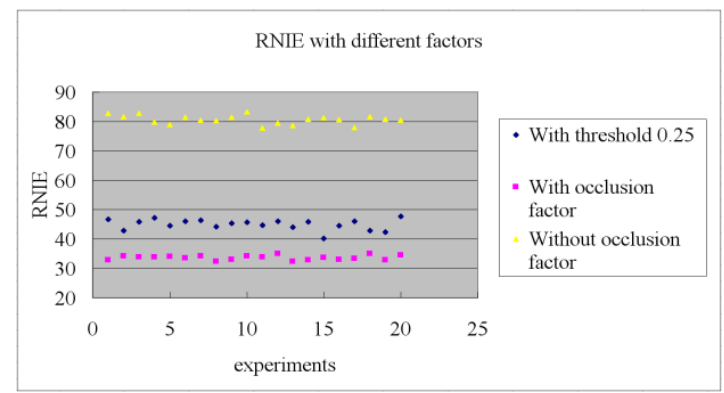

Fig. 5. RNIE of 20 experiments with different factors. (a) With threshold 0.25 . (b) With occlusion factor. (c) Without occlusion factor

\section{CONCLUSION AND FURTURE WORK}

This paper presents a quantitative measurement of information for vector field and the noise texture of it based on fuzzy feature measurement, which leads to a reasonable distribution of noise fragments. A noise generation algorithm is proposed based on the concepts mentioned above, and an approximate noise distribution is generated then. Experiments illustrate that a better visualization result with high information is obtained using noise generation algorithm proposed in this paper.
In future work, the efficiency of noise generation process needs to be enhanced. For noise space $512 * 512 * 512$ and 32768 noise fragments, a few seconds are needed to generate the whole noise texture, which is not fast enough for interactive visualization. It is important to apply this noise generation algorithm to $3 \mathrm{D}$ unsteady flow visualization. We will realize texture visualization for 3D unsteady vector field in the future.

\section{ACKNOWLEDGEMENT}

This work is financially supported by the National Grand Fundamental Research Program of China (Grant No.G2009CB723803), the National Natural Science Foundation of China (Grant No. 61170157), and the National Natural Science Foundation of China (Grant No. 60873120). Thanks for the support of visualization team of Room 621, School of Computer, National University of Defense Technology.

\section{REFERENCES}

[1] B. Cabral and L.C. Leedom (1993). Imaging Vector Fields Using Line Integral Convolution. In Proc. ACM SIGGRAPH '93, pp. 263-270.

[2] Xu Huaxun, Cheng Zhiquan, Ralph Martin and Li Sikun (2011). 3D Flow Feature Visualization via Fuzzy Measurement. The Visual Computer.

[3] Xu Huaxun, Li Sikun, Zeng Liang and Cai Xun (2011). Feature-based Adaptive Texture Visualization for Vector Field. Science in China Series F-Information Sciences.

[4] J.J. van Wijk (1991). Spot Noise-Texture Synthesis for Data Visualization. Computer Graphics (Proc. SIGGRAPH '91), Volume 25, pp. 309318.

[5] N. Max and B. Becker (1995). Flow Visualization Using Moving Textures. In Procceding of ICASW/LaRC Symp. Visualizing Time-Varying Data, pp. 77-87.

[6] B. Jobard, G. Erlebacher and M.Y. Hussaini (2002). Lagrangian-Eulerian Advection of Noise and Dye Textures for Unsteady Flow Visualization. IEEE Trans. Visualization and Computer Graphics, 8(3), pp. 211-222.

[7] J.J. van Wijk (2002). Image Based Flow Visualization. ACM Trans. Graphics, 21(3), pp. 745-754,.

[8] Han-Wei Shen and David L. Kao (1998). A New Line Integral Convolution Algorithm for Visualizing Time-Varying Flow Filed. IEEE Trans. Visualization and Computer Graphics, 4(2), pp. 98-108.

[9] A. Telea and J.J. van Wijk (2003). 3D IBFV: Hardware-Accelerated 3D Flow Visualization. In Proc. IEEE Visualization, pp. 233-240.

[10] D. Weiskopf, T. Schafhitzel and T. Ertl (2005). Real-Time Advection and Volumetric Illumination for the Visualization of 3D Unsteady Flow. In Proc. EG/IEEE VGTC Symp. Visualization (Eurovis '05), pp. 13-20.

[11] D. Weiskopf, T. Schafhitzel and T. Ertl (2007). Texture-Based Visualization of Unsteady 3D Flow by Real-Time Advection and Volumetric Illumination. IEEE Trans. Visualization and Computer Graphics, 13(3), pp. 569-582.

[12] M. Falk and D. Weiskopf (2008). Output-Sensitive 3D Line Integral Convolution. IEEE Transactions on visualization and computer graphics, 14(4), pp. 820-834.

[13] Helman J. and Hesselink L. (1989). Representation and display of vector field topology in fluid flow data sets. IEEE Computer, 22(8), pp. 27-36.

[14] Armin Pobitzer, Ronald Peikert, Raphael Fuchs, Benjamin Schindler, Alexander Kuhn, Holger Theisel, Krešimir Matkovi'c and Helwig Hauser (2010). On the Way towards Topology-Based Visualization of Unsteady Flow - the State of the Art. The State-Of-The-Art Proceeding of EUROGRAGHICS, pp. 137-154.

[15] J. Jeong and F. Hussain (1995). On the identification of a vortex. Journal of Fluid Mechanics, 28(5), pp. 69-94.

[16] S. Stegmaier, U. Rist and T.Ertl (2005). Opening the can of worms: An exploration tool for vortical flows. In Proceedings of IEEE Visualization pp. 463-470. 
[17] Haller G. (2005). An objective definition of a vortex. Journal of Fluid Mechanics, Volume 525, pp. 1-26.

[18] H. Janicke, A. Wiebel, G. Scheuermann and W.Kollmann (2007). Multifield Visualization Using Local Statistical Complexity. In Proceedings of IEEE Visualization, pp. 1384-1391.

[19] T. Schafhitzel, J.E. Vollrat, J.P. Gois, D. Weiskoph, A. Castelo and T. Ertl (2008). Topology-based Vortex Core Line Detection for Flow Visualization. Computer Graphics Forum, 27(3), pp. 1024-1030.

[20] P. Christoph, K. Jens, P. Steffen and H. Hanschristian (2009). Hierarchical Vortex Regions in Swirling Flow. Computer Graphics Forum, 28(1), pp. 863-870.

[21] Ming-Yuen Chan, Yingcai Wu and Huamin Qu (2007). Quality Enhancement of Direct Volume Rendered Images. Volume Graphics (The Eurographics Association), pp. 25-32.

[22] H. Niederreiter (1992). Random Number Generation and Quasi-Monte Carlo Methods. Society for Industrial and Applied Mathematics, Philadelphia, PA, USA.

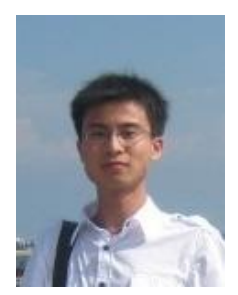

Huai-Hui Wang received his M.Sc. degree in Computer Science from the National University of Defense Technology in 2009. Currently, he is Ph.D. candidate at the College of Computer Science and Technology in the National University of Defense Technology. His research interests include texture-based visualization, feature-based visualization and unsteady vector field visualization.

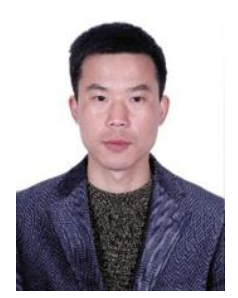

Hua-Xun Xu is a researcher in Army Aviation Institute of PLA, China, and a Ph.D. candidate in National University of Defense Technology. His current research interests include scientific visualization and virtual reality technologies.

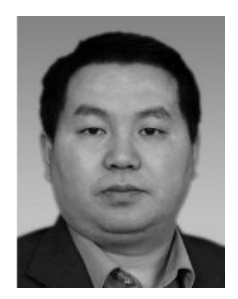

Liang Zeng, born in 1970, is an associate professor and senior member of China Computer Federation. His main research interests include parallel simulation, virtual reality and visualization.

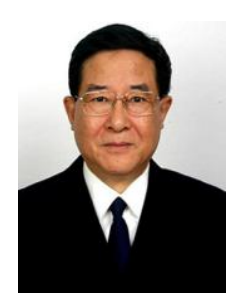

$\mathbf{S i - K u n ~} \mathbf{L i}$ is a professor at the College of Computer Science and Technology of the National University of Defense Technology. He is the principal of many national research projects of China such as the National Science Foundation program, the National High Technology Research and Development program and the National Basic Research program. His research interests include scientific visualization, virtual reality, very large scale integration, and the design of SoC. 